\title{
Riqueza de espécies de sub-bosque em um fragmento florestal urbano, Pernambuco, Brasil
}

\author{
Ana Cristina Ramos de Souza ${ }^{1}$ \\ Eduardo Bezerra de Almeida Jr. ${ }^{2}$ \\ Carmen Sílvia Zickel ${ }^{2 *}$ \\ ${ }^{1}$ Departamento de Biologia, Área Botânica, Faculdade São Lucas \\ Porto Velho - RO \\ ${ }^{2}$ Departamento de Biologia, Área Botânica \\ Universidade Federal Rural de Pernambuco, CEP 52171-900, Recife - PE, Brasil \\ *Autor para correspondência \\ zickelbr@yahoo.com
}

Submetido em 27/10/2008

Aceito para publicação em 25/05/2009

\section{Resumo}

Com o objetivo de caracterizar a composição florística do sub-bosque, o presente estudo foi desenvolvido no Parque Estadual de Dois Irmãos, $\left(08^{\circ} 01^{\prime} 15,1^{\prime \prime} \mathrm{S}\right.$ e $34^{\circ} 56^{\prime} 3,2^{\prime \prime} \mathrm{W}$ - sede), com área de aproximadamente 370ha, caracterizado como Floresta Ombrófila Densa das Terras Baixas, em que foram incluídos indivíduos de até 4,0m de altura, como arvoretas, arbusto, sub-arbustos e herbáceas terrícolas, em estádio fértil. As coletas quinzenais foram realizadas por um período de 24 meses. Foram coletadas 108 espécies, distribuídas em 86 gêneros e 49 famílias. As famílias com maior número de espécies foram Rubiaceae (14), Fabaceae (9), Melastomataceae (8), Asteraceae (8), Myrtaceae (6) e Poaceae (4). Fabaceae, Melastomataceae, Myrtaceae e Rubiaceae destacaram-se pelo maior número de espécies encontradas no sub-bosque do fragmento. De maneira geral, entre os levantamentos realizados em áreas de floresta atlântica de Pernambuco, constata-se a presença de um conjunto de espécies arbóreas comuns a essas florestas.

Unitermos: fragmento urbano, Pernambuco, riqueza, sub-bosque

\section{Abstract}

Understory species richness in an urban forest fragment, Pernambuco, Brazil. This study characterizes the floristic composition of the understory of Parque Estadual de Dois Irmãos, (0801'15.1' $\mathrm{S}$ and 34 $56^{\circ}$ '3.2”'W), an area of about 370 ha characterized as a lowland ombrophilous dense forest. The study included individuals with heights of up to $4.0 \mathrm{~m}$, such as treelets, shrubs, sub-bushes and terricolous herb plants, in fertile conditions. The collections were made every two weeks along a period of 24 months. A total of 108 species, belonging to 86 genera and 49 families, were recorded. The families with the highest number of species were Rubiaceae (14), Fabaceae (9) Melastomataceae (8), Asteraceae (8), Myrtaceae (6), and Poaceae (4). The Fabaceae, Melastomataceae, Myrtaceae and Rubiaceae presented the highest number of understory species in this fragment. Generally, among the studies made in the Atlantic forest areas in Pernambuco, the presence of a set of tree species common to these forests is evidenced.

Key words: Pernambuco, richness, understory, urban fragment 


\section{Introdução}

A floresta atlântica, embora considerada uma das regiões de maior biodiversidade do planeta, é um dos ecossistemas mais ameaçados do mundo (Peixoto e Gentry, 1990), comprometendo a biodiversidade, em relação a composição, estrutura e dinâmica. Mesmo assim, a floresta atlântica abriga mais de 8.000 espécies endêmicas de plantas vasculares, anfíbios, répteis, aves e mamíferos (Myers et al., 2000).

Algumas áreas de floresta atlântica encontram-se fragmentadas e próximas de grandes centros urbanos ou então estão envolvidas por vastas plantações de café, cana-de-açúcar e eucalipto (Morellato e Haddad, 2000), estando sujeitas a intervenções e constantes ameaças.

Em toda a região Nordeste do Brasil, existem apenas 2\% da floresta original (Ranta et al., 1998). Particularmente no estado de Pernambuco, a floresta atlântica ocupava aproximadamente $15,7 \%$ da área total, estendendo-se de norte a sul, desde a fronteira com a Paraíba até os limites com Alagoas (Andrade-Lima, 1960). Atualmente, resta cerca de $2 \%$ representado por um conjunto de fragmentos florestais, na maioria, com área inferior a 50ha (Ranta et al., 1998) e distribuídos em pequenos fragmentos (Silva e Tabarelli, 2000), quase não havendo grandes extensões florestais, como ainda se pode observar no Sul e Sudeste do Brasil (Brown Jr. e Brown, 1992; Capobianco e Lima, 1997).

Numa tentativa de conservação dos fragmentos da floresta atlântica em Pernambuco, através da lei no 9.989 (Pernambuco, 1987) foram criadas 40 reservas na Região Metropolitana do Recife. A Reserva Ecológica da Mata de Dois Irmãos merece atenção especial como um dos maiores fragmentos urbanos (FIDEM, 1987), e pode ser considerado um dos mais importantes laboratórios naturais do Nordeste (Tabarelli, 1998). Apesar da categoria de reserva ecológica, ainda é observada degradação nessa área que atualmente está inserida na categoria de Parque Estadual (Pernambuco, 1998).

Esses fragmentos florestais urbanos, circundados por uma matriz habitacional, abrigam em sua maioria testemunhos da flora arbórea da região e evidenciam a importância da sua conservação, aumentando assim o potencial educativo de fragmentos urbanizados (Cielo-
Filho e Santin, 2002). Alguns destes fragmentos são preciosos, pois contribuem para minimizar problemas causados pela superurbanização como a poluição do ar, impermeabilidade do solo, aquecimento do clima e outros fatores, além de atrair a fauna dispersora, especialmente pássaros, para a vegetação (Dislich e Pivello, 2002).

No entanto, em fragmentos de floresta atlântica, ainda são escassas informações sobre a flora do subbosque, devido ao maior enfoque dado ao estrato arbóreo, considerado como de maior riqueza de espécies (Kozera, 2001). Os estudos de Andreata et al. (1997) mostraram que existe uma grande diversidade de grupo de plantas não arbóreas presentes nos sub-bosques das florestas tropicais. No entanto, as espécies presentes neste grupo frequentemente são negligenciadas, mesmo sendo parte integrante e apresentando funções específicas na comunidade vegetal (Poulsen e Balslev, 1991). Alguns estudos enfatizando espécies do sub-bosque foram realizados por Bernacci (1992), Salis et al. (1996) e Andreata et al. (1997) mostrando a importância desse estrato na composição florística da área.

Pela importância deste fragmento florestal, este estudo teve como objetivo caracterizar a flora do subbosque, contribuindo com dados sobre a riqueza da área e comparação com a flora de sub-bosque de outras áreas de floresta atlântica para verificar sua diversidade.

\section{Material e Métodos}

\section{Área de estudo}

O Parque Estadual de Dois Irmãos localiza-se na Região Metropolitana do Recife, ocupando uma área de 370ha, constituindo um dos maiores fragmentos da floresta atlântica em perímetro urbano. Está localizado entre as coordenadas $08^{\circ} 01^{\prime} 15,1^{\prime \prime} \mathrm{S}$ e $34^{\circ} 56^{\prime} 3,2^{\prime \prime} \mathrm{W}-$ sede, com altitude entre 30 e $80 \mathrm{~m}$ e sua vegetação, de acordo com a classificação do IBGE (1992), é de Floresta Ombrófila Densa das Terras Baixas.

Apresenta solos do grupo Barreiras, originárias do Terciário, classificado como Latossolo VermelhoAmarelo distrófico (Jacomine et al., 1972), variando quanto à textura de arenoso a argiloso-arenoso, com 
pH de 4 a 5 (ácido). O clima é do tipo As' - tropical chuvoso, quente e úmido, com temperaturas médias mensais superiores a $23^{\circ} \mathrm{C}$, apresentando um período de alta umidade entre os meses de março e agosto, com precipitações máximas ocorrendo em junho e julho (Coutinho et al., 1998).

\section{Coleta florística}

Para definição da área de estudo foram escolhidos os pontos menos degradados para realização das coletas do material botânico. As excursões quinzenais à área foram realizadas no período de outubro de 1996 a maio de 1998. Nestas visitas foram percorridas todas as áreas escolhidas, sendo coletadas amostras de espécimes arbustivas, subarbustivas e ervas terrícolas em estádio reprodutivo ou com formação de esporos.

Para este estudo, foram considerados os seguintes conceitos: arvoreta, indivíduos com até $4 \mathrm{~m}$ de altura, com ramificação apenas na copa; arbustos, plantas com tamanho médio inferior a $4 \mathrm{~m}$, consistentes e lenhosos com tronco ramificado desde a base; subarbustos, plantas com até $1 \mathrm{~m}$ de altura (com base lenhosa e o restante herbáceo); ervas terrícolas, em geral com altura inferior a 1m, sem lignificação (Vidal e Vidal, 2000). Para esse estudo foram excluídas lianas, epífitas e trepadeiras herbáceas. Para verificar a ocorrência das espécies presentes no sub-bosque foram consultados alguns estudos de floresta atlântica realizados no estado de Pernambuco, desenvolvidos por Ferraz e Rodal (2006), Alves-Jr. et al. (2007), Rocha et al. (2008) e Silva Jr. et al. (2008). A identificação do material foi realizada com o auxílio de bibliografia especializada, chaves de identificação e por comparação com exsicatas do Herbário Prof. Vasconcelos Sobrinho (PEUFR) da Universidade Federal Rural de Pernambuco.

A listagem das espécies de angiospermas do sub-bosque seguiu a proposta de classificação das famílias reconhecidas pelo APG II (2003) e para as pteridófitas seguiu a proposta de Smith et al. (2006). Para uniformização dos nomes dos autores foi utilizado Brummitt e Powell (1992).

\section{Resultados e Discussão}

Foram identificadas 108 espécies de 86 gêneros, distribuídas em 49 famílias (Tabela 1). As famílias com maior número de espécies foram Rubiaceae (14 espécies), Fabaceae (9), Melastomataceae (8), Asteraceae (8), Myrtaceae (4) e Poaceae (4), perfazendo 43,5\% do total de espécies. Estas famílias são representativas para as áreas de floresta atlântica (Mori et al., 1983), representadas por espécies arbustivas e ervas terrícolas como no presente estudo, até espécies arbóreas, compondo estratos diferenciados das florestas (Siqueira et al., 2001; Ferraz e Rodal, 2006; Rodal e Nascimento, 2006). Tabarelli e Mantovani (1997) apontam que Myrtaceae, Rubiaceae e Melastomataceae se destacam pelo maior número de espécies no sub-bosque em floresta atlântica, principalmente com os gêneros Psidium, Psychotria e Miconia.

Os gêneros mais representativos foram Psychotria (6 spp), Miconia (4), Inga (4), Erythroxylum (3), Piper (3), e Casearia, Cordia, Diodia, Ichnanthus, Myrcia, Rhynchospora e Solanum, com duas espécies cada.

A família Rubiaceae apresentou maior expressividade e maior número de espécies na área, com destaque para o gênero Psychotria. Tanto a família quanto o gênero estão entre os mais abundantes no sub-bosque de áreas de florestas neotropicais (Gentry e Emmons, 1987) e de floresta atlântica (Joly et al., 1991; Negrelle, 2006). No entanto, à medida que as florestas vão diminuindo a disponibilidade de água, indivíduos sub-arbusivos e/ou arbustivos como Psychotria, por exemplo, tornam-se menos comuns, sendo observados com maior frequência indivíduos arbóreos ou pequenas arvoretas como Guettarda, Randia e Tocoyena (Tavares et al., 1969; Ferraz et al., 1998; Rodal e Nascimento, 2002). 
TABELA 1: Lista das espécies do sub-bosque no Parque Estadual de Dois Irmãos, Recife, Pernambuco, com os respectivos números de coletores, hábitos: erva terrícola $(\mathrm{Et})$, subarbusto $(\mathrm{Sb})$, arbusto $(\mathrm{Ab})$, arvoreta (Av) e nome dos especialistas de algumas famílias.

\begin{tabular}{|c|c|c|}
\hline Famílias/ Espécies & Coletor/ Número & Hábito \\
\hline \multicolumn{3}{|l|}{ Acanthaceae } \\
\hline Dicliptera ciliaris Juss. & A.C.Souza, 157 & $\mathrm{Sb}$ \\
\hline Nelsonia brunelloides (Lam.) Kuntze & A.C.Souza, 124 & Et \\
\hline \multicolumn{3}{|l|}{ Anacardiaceae } \\
\hline Tapirira guianensis Aubl. & A.C.Souza, 61 & Av \\
\hline Thyrsodium schomburgkianum Benth. & F.F.Melo, 52 & $\mathrm{Av}$ \\
\hline \multicolumn{3}{|l|}{ Annonaceae } \\
\hline Cymbopetalum brasiliense (Vell.) Benth. ex Baill. & A.C.Souza, 65 & $\mathrm{Ab}$ \\
\hline Guatteria australis A. St-Hil. & A.C.Souza, 36 & $\mathrm{Ab}$ \\
\hline \multicolumn{3}{|l|}{ Apocynaceae } \\
\hline Himatanthus phagedaenicus (Mart.) Woodson & F.F.Melo, 51 & Av \\
\hline \multicolumn{3}{|l|}{ Arecaceae } \\
\hline Bactris humilis (Wallace) Burret & J.R.Cantarelli, 23 & $\mathrm{Ab}$ \\
\hline \multicolumn{3}{|l|}{ Asteraceae - (Roseli Barros-UFPI; Rita Pereira-IPA) } \\
\hline Conyza bonariensis (L.) Cronquist & V.Santos, 14 & Et \\
\hline Elephantopus mollis Kunth & A.C.Souza, 167 & Et \\
\hline Emilia sagitatta (Vahl) DC. & A.C.Souza, 189 & Et \\
\hline Eupatorium ballotifolium Kunth. & F.F.Melo, 28 & $\mathrm{Sb}$ \\
\hline Pluchea sagittalis (Lam.) Cabrera & J.B.Accioly, 179 & Et \\
\hline Porophyllum ruderale (Jacq.) Cass. & V.Santos, 11 & $\mathrm{Sb}$ \\
\hline Rolandra argentea Rottb. & A.C.Souza, 166 & $\mathrm{Sb}$ \\
\hline Vernonia brasiliana (L.) Druce & A.C.Souza, 35 & $\mathrm{Ab}$ \\
\hline \multicolumn{3}{|l|}{ Boraginaceae (J.Iranildo Melo-UFPB) } \\
\hline Cordia multispicata Cham. & A.C.Souza, 50 & $\mathrm{Ab}$ \\
\hline Cordia nodosa Lam. & A.C.Souza, 33 & $\mathrm{Ab}$ \\
\hline \multicolumn{3}{|l|}{ Burseraceae } \\
\hline Protium heptaphyllum (Aubl.) March. & S.S.Lira, s/n & $\mathrm{Av}$ \\
\hline \multicolumn{3}{|l|}{ Cannabaceae } \\
\hline Trema micrantha (L.) Blume & A.C.Souza, 62 & $\mathrm{Ab}$ \\
\hline \multicolumn{3}{|l|}{ Celastraceae } \\
\hline Maytenus distichopylla Mart. ex Reissek & A.C.Souza, 279 & $\mathrm{Ab}$ \\
\hline \multicolumn{3}{|l|}{ Chrysobalanaceae } \\
\hline Hirtella racemosa Lam. & F.F.Melo, 13 & $\mathrm{Ab}$ \\
\hline \multicolumn{3}{|l|}{ Clusiaceae } \\
\hline Clusia nemorosa G. Mey & F.F.Melo, 47 & $\mathrm{Av}$ \\
\hline \multicolumn{3}{|l|}{ Commelinaceae (Roxana Barreto-UFPE) } \\
\hline Commelina rufipes var. glabrata (D.R.Hunt) Faden \& D.R.Hunt & A.C.Souza, 450 & Et \\
\hline \multicolumn{3}{|l|}{ Connaraceae } \\
\hline Connarus suberosus Planch. & F.F.Melo, 25 & $\mathrm{Ab}$ \\
\hline \multicolumn{3}{|l|}{ Costaceae } \\
\hline Costus spiralis (Jacq.) Roscoe & A.C.Souza, 105 & Et \\
\hline \multicolumn{3}{|l|}{ Cyperaceae (Marccus Alves-UFPE) } \\
\hline Cyperus laxus Lam. & A.C.Souza, 313 & Et \\
\hline
\end{tabular}


Rhynchospora cephalotes (L.) Vahl

A.C.Souza, 330

Et

Rhynchospora comata (Link.) Roem. \& Schult.

A.C.Souza, 331

Et

Erythroxylaceae (M. Iracema Loiola-UFC)

Erythroxylum citrifolium A. St-Hil.

A.C.Souza, $60 \quad$ Ab

Erythroxylum mucronatum Benth.

A.C.Souza, 347

$\mathrm{Ab}$

Erythroxylum squamatum Sw.

A.C.Souza, 341

$\mathrm{Ab}$

Euphorbiaceae (Maria de Fátima Lucena-UFPE)

Chaetocarpus myrsinites Baill.

A.C.Souza, $204 \quad \mathrm{Ab}$

Gymnanthes verticillata (Klotszch) Webster

A.C.Souza, 226

$\mathrm{Ab}$

Pogonophora schomburgkiana Miers ex Benth.

A.C.Souza, 222

$\mathrm{Ab}$

Fabaceae: Caesalpinioideae (J. Elias Lima-PEUFR)

Chamaecrista nictitans (L.) Moench.

V.Santos, 13

$\mathrm{Ab}$

Senna georgica H.S.Irwin \& Barneby

A.C.Souza, 173

Av

Fabaceae: Faboideae (J.Y.Tamashiro-UNICAMP)

Bocoa limae R.S.Cowan

A.C.Souza, $198 \quad$ Av

Crotalaria stipularia Desv.

A.C.Souza, $24 \quad \mathrm{Sb}$

Swartzia pickelii Killip ex Ducke

F.F.Melo, 49

Av

Fabaceae: Mimosoideae (Flávia Garcia-UFV)

Inga capitata Desv.

A.C.Souza, $373 \quad A b$

Inga laurina (Sw.) Willd.

F.F.Melo, 45

Av

Inga thibaudiana DC.

F.F.Melo, 48

Av

Inga vera (Benth.) T.D. Penn

A.C.Souza, 45

Av

Gentianaceae

Irlbachia alata (Aubl.) Maas

A.C.Souza, 13

Et

Heliconiaceae

Heliconia psittacorum Sessé \& Moc.

A.C.Souza, 22

Et

Hypericaceae

Vismia guianensis (Aubl.) Choisy

A.C.Souza, 47

Av

Lauraceae

Ocotea glomerata (Nees) Mez

A.C.Souza, 382

Av

Lindsaeaceae (Iva C.L. Barros-UFPE)

Lindsaea lancea (L.) Bedd.

A.C.Souza, 393

Et

Lycopodiaceae (Iva C.L. Barros-UFPE)

Lycopodiella cernua (L.) Pich. Serm.

A.C.Souza, 17

Et

Lythraceae

Cuphea carthagenensis (Jacq.) J.F. Macbr.

F.Lucena, 289

$\mathrm{Sb}$

Malpighiaceae

Byrsonima sericea DC.

A.C.Souza, 174

Av

Malvaceae (G.S.Baracho-UFPE; M.Tschá -PEUFR)

Sida linifolia Cav.

A.C.Souza, 190

Triumfetta althaeoides Lam.

F.F.Melo, 32

$\mathrm{Sb}$

Marantaceae (Karla N. Arns - PEUFR)

Ischnosiphon gracilis Korn

A.C.Souza, 348

Et

Stromanthe porteana A. Griseb

A.C.Souza, 12

Et

Melastomataceae (Renato Goldenberg-UFPR)

Clidemia hirta (L.) Don.

A.C.Souza, 63

$\mathrm{Ab}$

Henriettea succosa (Aubl) DC.

A.C.Souza, 303

Av 
Leandra sp.

A.C.Souza, 111

$\mathrm{Ab}$

Miconia albicans (Sw.) Triana

A.C.Souza, 59

$\mathrm{Ab}$

Miconia ciliata (Rich.) DC.

A.C.Souza, 37

$\mathrm{Ab}$

Miconia cuspidata Naud.

A.C.Souza, 40

$\mathrm{Ab}$

Miconia prasina (Sw.) DC.

A.C.Souza, 287

$\mathrm{Ab}$

Pterolepis polygonoides (DC.) Triana

A.C.Souza, 27

Et

Moraceae

Sorocea hilarii Gaudich.

A.C.Souza, 402

Av

Myrsinaceae

Cybianthus detergens Mart.

F.F.Melo, 53

$\mathrm{Ab}$

Myrtaceae (Graziela M. Barroso-HB)

Eugenia hirta O. Berg.

F.F.Melo, 20

$\mathrm{Ab}$

Gomidesia spectabilis (DC.) O. Berg.

A.C.Souza, 11

$\mathrm{Ab}$

Myrcia crassifolia Kiaersk

A.C.Souza, 15

$\mathrm{Ab}$

Myrcia lancea (O. Berg.) Mattos

F.F.Melo, 63

$\mathrm{Ab}$

Nyctaginaceae

Guapira opposita (Vell.) Reitz

A.Laurênio, 520

Av

Ochnaceae

Ouratea polygyna Engler

A.C.Souza, 163

$\mathrm{Ab}$

Orchidaceae (Leonardo P. Félix-UFPB)

Prescottia stachyodes Lindl.

A.C.Souza, 439

Et

Sarcoglottis grandiflora (Hook.) Klotzsch

J.Cantarelli, 29

Et

Picramniaceae

Picramnia gardneri Planch.

A.C.Souza, 69

$\mathrm{Ab}$

Piperaceae (Elsie Guimarães-RB)

Piper aduncum L.

A.C.Souza, $21 \quad \mathrm{Sb}$

Piper arboreum Aubl.

A.C.Souza, $97 \quad \mathrm{Sb}$

Piper marginatum Jacq.

A.C.Souza, 284

$\mathrm{Sb}$

Poaceae (Marcus Alves-UFPE)

Ichnanthus nemoralis (Schrad. ex Roem. \& Schult.) Hitch. \& Chase

A.C.Souza, 115

Et

Ichnanthus pallens (Sw.) Munro

A.C.Souza, 370

Et

Olyra latifolia L.

A.C.Souza, $79 \quad$ Et

Sporobolus virginicus (L.) Kunth.

A.C.Souza, 308

Et

Polygalaceae

Polygala martiana A.W. Bennett

A.C.Souza, 208

Et

Polygonaceae

Coccoloba guianensis Meisn.

A.Laurênio, 498

$\mathrm{Ab}$

Pteridaceae (Iva C.L. Barros-UFPE)

Adiantum latifolium Lam.

A.C.Souza, 15

Et

Rubiaceae (Daniela Zappi-KEW)

Chomelia tristis Müll Arg.

A.C.Souza, 447

$\mathrm{Ab}$

Diodia ocymifolia (Willd.) Bremek.

A.C.Souza, 14

Et

Diodia sarmentosa $\mathrm{Sw}$.

A.C.Souza, 458

Et

Mitracarpus lhotzkyanus Cham.

A.C.Souza, 14

Et

Palicourea crocea (Sw.) Roem. \& Schult.

A.C.Souza, 56

$\mathrm{Ab}$

Posoqueria latifolia Roem. \& Schult.

A.C.Souza, 98

$\mathrm{Ab}$

Psychotria bracteocardia (Benth. \& Hooker) Steyerm.

A.C.Souza, 306

$\mathrm{Ab}$ 
Psychotria capitata Ruiz \& Pav.

A.C.Souza, $67 \quad \mathrm{Ab}$

Psychotria carthagenensis Jacq.

A.C.Souza, 19

$\mathrm{Ab}$

Psychotria erecta (Aubl.) Standley \& Steyerm.

A.C.Souza, $401 \quad \mathrm{Ab}$

Psychotria hoffmannseggiana (Roem. \& Schult.) Müll. Arg.

A.C.Souza, 18

$\mathrm{Ab}$

Psychotria martiana Müll. Arg

A.C.Souza, 70

$\mathrm{Ab}$

Spermacoce scabiosoides (Cham. \& Schltdl.) Kuntze

A.C.Souza, 158

$\mathrm{Et}$

Staelia aurea K. Schum.

F.F.Melo, 21

Et

\begin{tabular}{|c|c|c|}
\hline \multicolumn{3}{|l|}{ Salicaceae } \\
\hline Casearia commersoniana Cambess. & A.C.Souza, 185 & $\mathrm{Av}$ \\
\hline Casearia javitensis Kunth & A.C.Souza, 280 & $\mathrm{Av}$ \\
\hline \multicolumn{3}{|l|}{ Sapindaceae (Genise Somner-RB) } \\
\hline Cupania racemosa (Vell) Radlk. & F.F.Melo, 74 & $\mathrm{Av}$ \\
\hline \multicolumn{3}{|l|}{ Siparunaceae } \\
\hline Siparuna guianensis Aubl. & A.C.Souza, 275 & $\mathrm{Ab}$ \\
\hline \multicolumn{3}{|l|}{ Solanaceae } \\
\hline Brunfelsia uniflora (Pohl) D.Don & A.C.Souza, 346 & $\mathrm{Ab}$ \\
\hline Solanum gracillimum Sendtn. & A.C.Souza, 221 & $\mathrm{Ab}$ \\
\hline Solanum stramoniifolium Jacq. & A.C.Souza, 26 & $\mathrm{Ab}$ \\
\hline \multicolumn{3}{|l|}{ Verbenaceae } \\
\hline Stachytarpheta cayennensis (Rich.) Vahl. & A.C.Souza, 387 & Et \\
\hline \multicolumn{3}{|l|}{ Violaceae } \\
\hline Paypayrola blanchetiana Tul. & A.C.Souza, 299 & $\mathrm{Av}$ \\
\hline
\end{tabular}

Miconia foi outro gênero bem representado no levantamento florístico, pois do total de oito espécies da família Melastomataceae, quatro pertencem ao gênero Miconia. Essas espécies foram observadas com maior intensidade ao longo das trilhas e nas clareiras naturais. Vicente (1999) observou que nos levantamentos realizados no Nordeste, Melastomataceae se destaca entre as famílias que apresentam maior número de espécies por ser uma planta pioneira e contribuir para a renovação e dinâmica das espécies vegetais quando da perda de indivíduos mais velhos, uma vez que colonizam rapidamente as áreas (Pizatto, 1999; Werneck et al., 2000; Armelin e Mantovani, 2001). No entanto, essa facilidade em colonizar áreas abertas, clareiras ou bordas de fragmento, pode levar algumas famílias como Asteraceae, Poaceae, Solanaceae e Melastomataceae a serem apontadas como grupos indicadores de ambientes alterados (Teixeira e Mantovani, 1998; Tabarelli e Mantovani, 1999).

Pedralli et al. (1997) relacionaram a riqueza de Asteraceae às espécies herbáceas invasoras, por terem facilidade de propagação e por indicarem estádios iniciais de sucessão devido à rapidez na colonização das áreas.
De maneira geral, entre os levantamentos realizados em áreas de floresta atlântica no estado de Pernambuco, constata-se a presença de um grupo de espécies comuns no sub-bosque, como: Ageratum conyzoides, Borreria verticillata, Casearia javitensis, Costus spiralis, Maytenus distichopylla, Psychotria carthagenensis, Solanum paludosum e Stylosanthes scabra (Silva, 2004). Contudo, analisando três áreas de floresta atlântica, Zickel et al. (2008) mostraram que o conjunto de espécies do sub-bosque não apresentou alta similaridade florística entre as áreas, apontando indícios de alta diversidade e riqueza de espécies para esse estrato nos diferentes fragmentos.

Por outro lado, essa riqueza pode estar sendo favorecida pelos possíveis distúrbios que tenham ocorrido na área, visto que com o corte indiscriminado aumenta a entrada de luminosidade e facilita o desenvolvimento de espécies intolerantes à sombra (Vieira e Hosokawa, 1989) comprometendo assim a composição florística original e, portanto, a dominância local das espécies (Vidal et al., 1998; Werneck et al., 2000). Podendo ocasionar uma descaracterização do 
estrado devido à entrada de espécies ruderais ou de ampla distribuição.

No presente estudo, as espécies listadas na área também são registradas em áreas de Florestas das Terras Baixas, Estacionais e Montanas (Melo e Rodal, 2003; Ferraz e Rodal, 2006; Alves-Jr. et al., 2007; Rocha et al., 2008). Porém, espécies de menor porte que compõem o sub-bosque não são registradas, porque a maioria dos trabalhos enfatiza apenas o componente arbóreo (Zickel, 1995), não considerando ou dando menor importância à vegetação do sub-bosque.

Foram registradas na área quatro formas biológicas, representados por ervas, subarbustos, arbustos e arvoretas (Figura 1). As 33 espécies ervas terrícolas amostradas foram encontradas em maior abundância onde havia maior incidência luminosa, diferentemente da floresta na Serra de Itabaiana (Vicente, 1999), onde a riqueza específica das ervas foi menor (13). Esse fato pode acontecer naturalmente por haver um número limitado de espécies ervas terrícolas no interior de florestas devido às condições ambientais peculiares a que estão submetidas (Mantovani, 1987; Citadini-Zanette e Baptista, 2008; Palma et al., 2008). Já as espécies arbustivas e subarbustivas, somadas, representaram quase $50 \%$ das espécies do sub-bosque, o que demonstra tolerância de muitas espécies ao sombreamento e a importância desses hábitos na composição da floresta atlântica. Além disso, quase $20 \%$ dos indivíduos jovens de árvores foram coletadas neste estrato, apontando a necessidade de mais estudos sobre o sub-bosque.

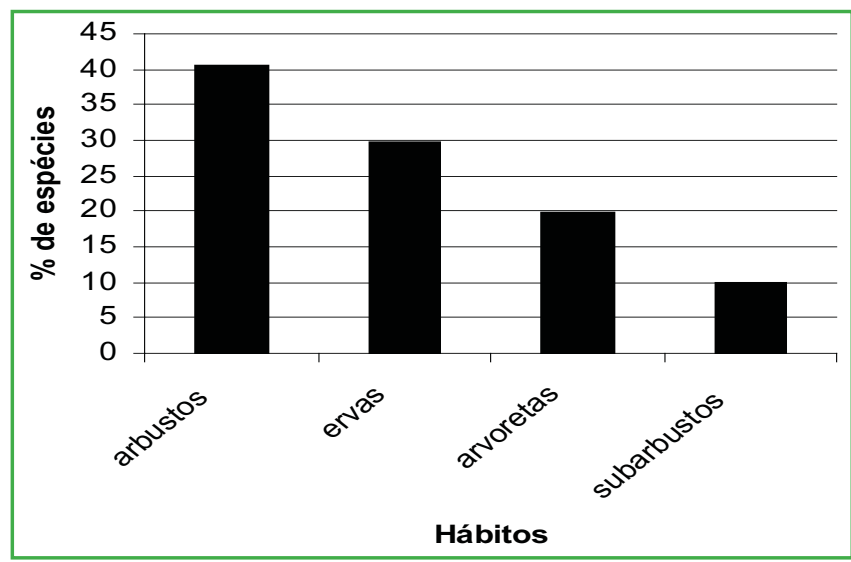

FIGURA 1: Distribuição do número de espécies por hábito da flora do sub-bosque do Parque Estadual de Dois Irmãos, Recife, Pernambuco.
Quanto ao estrato arbóreo, Guedes (1998) já havia apontado, para o Parque Estadual de Dois Irmãos, que as espécies emergentes apresentavam indivíduos que ultrapassavam $31 \mathrm{~m}$ de altura e que o estrato arbustivo e o herbáceo eram constituídos por um baixo número de espécies. No entanto, os dados encontrados neste estudo, por estar direcionado para o sub-bosque, apontaram $40,5 \%$ de espécies arbustivas e $29,8 \%$ de ervas terrícolas, demonstrando a riqueza florística do sub-boque, devido principalmente aos fatores abióticos diferenciados observados no interior do fragmento.

Apesar deste fragmento florestal localizar-se no centro urbano da cidade de Recife, o desenvolvimento das espécies e o sucesso reprodutivo está diretamente relacionado com a permanência dos polinizadores na área (Teixeira e Machado, 2004), mostrando também a importância da conservação da fauna local. Além disso, Mantovani (1987) e Zickel (1995) relataram que há uma expectativa de valorização do sub-bosque, já que ele constitui um banco genético com grande número de espécies e, particularmente, com grande variedade de formas de vida. Logo, as espécies do sub-bosque contribuem substancialmente para a manutenção das populações de animais polinizadores e dispersores, reforçando a necessidade de proteção de áreas remanescentes e de fragmentos urbanos do Estado.

\section{Referências}

Alves-Jr., F. T.; Brandão, F. L. S. B.; Rocha, K. D.; Silva, J. T.; Marangon, L. C.; Ferreira, R. L. C. 2007. Estrutura diamétrica e hipsométrica do componente arbóreo de um fragmento de mata atlântica, Recife-PE. Cerne, 13 (1): 83-95.

Andrade-Lima, D. 1960. Tipos de florestas de Pernambuco. Anais da Associação dos Geógrafos Brasileiros, 12: 69-85.

Andreata, R. H. P.; Gomes, M.; Baumgratz, J. F. A. 1997. Plantas herbáceo-arbustivas terrestres da Reserva Ecológica de Macaé de Cima. In: Lima, H. C. \& Guedes-Bruni, R. R. (Eds). Serra de Macaé de Cima: Diversidade florística e conservação em Mata Atlântica. Instituto de Pesquisas Jardim Botânico do Rio de Janeiro, Rio de Janeiro, Brasil, p.65-73.

APG II - The Angiosperm Phylogeny Group. 2003. An update of the Angiosperm Phylogeny Group classification for the orders and families of flowering plants: APG II. Botanical Journal of the Linnean Society, 141: 399-436.

Armelin, R. S.; Mantovani, W. 2001. Definições de clareira natural e suas implicações no estudo da dinâmica sucessional em florestas. Rodriguésia, 52 (81): 5-15. 
Bernacci, L. C. 1992. Estudo florístico e fitossociológico de uma floresta no município de Campinas, com ênfase nos componentes herbáceo e arbustivo. Dissertação de Mestrado, Universidade Estadual de Campinas, Brasil, 147pp.

Brown Jr., K. S.; Brown, G. G. 1992. Habitat alteration and species loss in Brazilian forests. In: Whitmore, T. C. \& Sayer, J. A. (Eds) Tropical deforestation and species extinction. Chapman \& Hall, London, UK, p.129-142.

Brumitt, R. K.; Powell, C. E. 1992. Authors of plant names. Royal Botanic Gardens, Kew, UK, 732pp.

Capobianco, J. P. R.; Lima, A. 1997. A evolução da proteção legal da Mata Atlântica. In: Lima, A. \& Capobianco, J. P. R. (Orgs). Mata Atlântica: Avanços legais e institucionais para sua conservação. Documentos do ISA $n^{\circ}$ 4. Instituto Socioambiental, São Paulo, Brasil, p.7-18.

Cielo-Filho, R.; Santin, D. A. 2002. Estudo florístico e fitossociológico de um fragmento florestal urbano - Bosque dos Alemães, Campinas, SP. Revista Brasileira de Botânica, 25 (3): 291-301.

Citadini-Zanette, V.; Baptista, L. R M. 1989. Vegetação herbácea terrícola de uma comunidade florestal em Limoeiro, município de Torres, Rio Grande do Sul, Brasil. Boletim do Instituto de Biociências, 45: 1-87.

Coutinho, R. Q.; Lima, M. F.; Neto, J. B. S.; Silva, E. P. 1998. Características climáticas, geológicas, geomorfológicas e geotécnicas da Reserva Ecológica de Dois Irmãos. In: Machado, I. C.; Lopes, A. V. \& Porto, K. C. (Eds). Reserva Ecológica de Dois Irmãos: Estudos em um remanescente de Mata Atlântica em área urbana. SECTMA, UFPE, Recife, Brasil, p.21-50.

Dislich, R.; Pivello, V. R. 2002. Tree structure and species composition changes in an urban tropical forest fragment (São Paulo, Brazil) during a five-year interval. Boletim de Botânica da Universidade de São Paulo, 20: 1-12.

Ferraz, E. M. N.; Rodal, M. J. N. 2006. Caracterização fisionômicaestrutural de um remanescente de Floresta Ombrófila Montana de Pernambuco, Brasil. Acta Botanica Brasilica, 20 (4): 911-926.

Ferraz, E. M. N.; Rodal, M. J. N.; Sampaio, E. V. S. B.; Pereira, R. C. A. 1998. Composição florística em trechos de vegetação de caatinga e brejo de altitude na região do Vale do Pajeú, Pernambuco. Revista Brasileira de Botânica, 21 (1): 7-15.

FIDEM. 1987. Reservas ecológicas da Região Metropolitana do Recife. Série de Desenvolvimento Urbano e Meio Ambiente, Recife, Brasil, 108pp.

Gentry, A. H.; Emmons, L. H. 1987. Geographical variation in fertility, phenology, and composition of the understory of neotropical forests. Biotropica, 19: 216-227.

Guedes, M. L. S. 1998. A vegetação fanerogâmica da Reserva Ecológica de Dois Irmãos. In: Machado, I. C.; Lopes, A. V. \& Porto, K. C. (Eds). Reserva Ecológica de Dois Irmãos: Estudos em um remanescente de Mata Atlântica em área urbana. SECTMA, UFPE, Recife, Brasil, p.157-172.

IBGE. 1992. Manual técnico da vegetação brasileira. IBGE, Rio de Janeiro, Brasil, 92pp.

Jacomine, P. K. T.; Cavalcanti, A. C.; Burgos, N.; Pessoa, S. C. P.; Silveira, C. O. 1972. Levantamento exploratório Reconhecimento de solos do estado de Pernambuco. DPP/ SUDENE, Recife, Brasil, 359pp.
Joly, A. B.; Leitão-Filho, H. F.; Silva, S. M. 1991. O patrimônio florístico. In: Câmara, I. G. (Ed.). Mata Attântica. Editora Indx e Fundação SOS Mata Atlântica, São Paulo, Brasil, p.96-128.

Kozera, C. 2001. Composição florística e estrutura fitossociológica do estrato herbáceo-subarbustivo em duas áreas de floresta ombrófila densa, Paraná, Brasil. Tese de Doutorado, Universidade Estadual de Campinas, Brasil, 175pp.

Mantovani, W. 1987. Análise florística fitossociológica do estrato herbáceo-subarbustivo do cerrado na Reserva Biológica de Mogi-Guaçu e em Itirapina, SP. Tese de Doutorado, Universidade Estadual de Campinas, Brasil, 203pp.

Melo, J. I. M.; Rodal, M. J. N. 2003. Levantamento florístico de um trecho de floresta serrana no planalto de Garanhuns, Estado de Pernambuco. Acta Scientiarum: Biological Sciences, 25 (1): 173178.

Morellato, L. P. C.; Haddad, C. F. B. 2000. Introduction: The Brazilian Atlantic Forest. Biotropica, 32: 786-792.

Mori, S. A.; Boom, B. M.; Carvalho, A. M.; Santos, T. S. 1983. Southern Bahian moist forest. The Botanical Review, 49 (2): 155-232.

Myers, N.; Mittermeier, R. A.; Mittermeier, C. G.; Fonseca, G. A. B.; Kent, J. 2000. Biodiversity hotspots for conservation priorities. Nature, 403: 853-858.

Negrelle, R. R. B. 2006. Composição florística e estrutura vertical de um trecho de Floresta Ombrófila Densa de Planície Quaternária. Hoehnea, 33 (3): 261-289.

Palma, C. B.; Inácio, C. D.; Jarenkow, J. A. 2008. Florística e estrutura da sinúsia herbácea terrícola de uma floresta estacional de encosta no Parque Estadual de Itapuã, Viamão, Rio Grande do Sul, Brasil. Revista Brasileira de Biociências, 6 (3): 151-158.

Pedralli, G.; Freitas, V. L. O.; Meyer, S. T.; Teixeira, M. C. B.; Gonçalves, A. P. S. 1997. Levantamento florístico na Estação Ecológica do Tuipuí, Ouro Preto, MG. Acta Botanica Brasilica, 11 (2): 119-213.

Peixoto, A. L.; Gentry, A. 1990. Diversidade e composição florística da mata de tabuleiro na Reserva Florestal de Linhares (ES, Brasil). Revista Brasileira de Botânica, 13: 19-25.

Pernambuco. 1987. Lei ${ }^{\circ} 9989$, de 13 de janeiro de 1987. Define as reservas ecológicas da região metropolitana do Recife. Diário Oficial do Estado de Pernambuco, Recife, 14 de janeiro de 1987. Pernambuco. 1998. Lei n ${ }^{\circ} 11622$, de 29 de dezembro de 1998. Parque Estadual de Dois Irmãos. Diário Oficial do Estado de Pernambuco, Recife, 30 de dezembro de 1998.

Pizatto, W. 1999. Avaliação biométrica da estrutura e da dinâmica de uma floresta ombrófila mista em São João do Triunfo-PR: 1995 a 1998. Dissertação de Mestrado, Universidade Federal do Paraná, Brasil, 172pp.

Poulsen, A. D.; Balslev, H. 1991. Abundance and cover of ground herbs in an Amazonia rain forest. Journal of Vegetation Science, 2: 315-322.

Ranta, P.; Brom, T.; Joensuu, E.; Mikko, S. 1998. The fragmented atlantic forest of Brazil: size, shape and distribution of forest fragments. Biodiversity Conservation, 7: 385-403.

Rocha, K. D.; Chaves, L. F. C.; Marangon, L. C.; Lins e Silva, A. C. B. 2008. Caracterização da vegetação arbórea adulta em um fragmento de floresta atlântica, Igarassu, PE. Revista Brasileira de Ciências Agrárias, 3 (1): 35-41. 
Rodal, M. J. N.; Nascimento, L. M. 2002. Levantamento florístico da floresta serrana da Reserva Biológica de Serra Negra, microrregião de Itaparica, Pernambuco, Brasil. Acta Botanica Brasilica, 16 (4): 481-500.

Rodal, M. J. N.; Nascimento, L. M. 2006. The arboreal component of a dry forest in Northesttern, Brazil. Brazilian Journal of Biology, 66 (2A): 479-491.

Salis, S. M.; Zickel, C. S.; Tamashiro, J. Y. 1996. Fitossociologia do sub-bosque da mata da Reserva de Santa Genebra, Campinas (estado de São Paulo). Naturalia, 21: 171-180.

Silva, I. M. M. S. 2004. Riqueza e fisionomia do sub-bosque em fragmento de Floresta Ombrófila de Terras Baixas, Pernambuco. Monografia de Graduação, Universidade Federal Rural de Pernambuco, Recife, Brasil, 78pp.

Silva, J. M. C.; Tabarelli, M. 2000. Tree species impoverishment and the future flora of the Atlantic forest of northeast Brazil. Nature, 404: 72-74.

Silva Jr., J. F.; Marangon, L. C.; Ferreira, R. L. C.; Feliciano, A. L. P.; Brandão, C. F. L. S.; Alves Jr., F. T. 2008. Fitossociologia do componente arbóreo em um remanescente de floresta atlântica no município do Cabo de Santo Agostinho, PE. Revista Brasileira de Ciências Agrárias, 3 (3): 276-282.

Siqueira, D. R.; Rodal, M. J. N.; Lins e Silva, A. C. B.; Melo, A. L. 2001. Physionomy, structure and floristic in area of Atlantic Forest in northeast Brasil. In: Gottsberger, G. \& Liede, S. (Eds). Life forms and dynamics in tropical forests. Gebr. Borntraeger Verlagsbuchhandlung, Berlim-Stuttgart, Germany, p.11-27.

Smith, A. R.; Pryer, K. M.; Schuettpelz, E.; Korall, P.; Schneider, H.; Wolf, P. G. 2006. A classification for extant ferns. Taxon, 55 (3): 705-731.

Tabarelli, M. 1998. Dois Irmãos: O desafio da conservação Biológica em um fragmento de Floresta Tropical. In: Machado, I. C.; Lopes, A. V. \& Porto, K. C. (Eds). Reserva Ecológica de Dois Irmãos: Estudos em um remanescente de Mata Atlântica em área urbana. SECTMA, UFPE, Recife, Brasil, p.311-323.

Tabarelli, M.; Mantovani, W. 1997. Colonização de clareiras naturais na floresta atlântica no sudeste do Brasil. Revista Brasileira de Botânica, 20 (1): 57-66.

Tabarelli, M.; Mantovani, W. 1999. Clareiras naturais e a riqueza de espécies pioneiras em uma floresta atlântica montana. Revista Brasileira de Biologia, 59 (2): 251-261.
Tavares, S.; Paiva, F. A. V.; Tavares, E. J. S.; Lima, J. L. S.; Carvalho, G. H. 1969. Inventário florestal de Pernambuco. Estudo preliminar das matas remanescentes do município de São José do Belmonte. Boletim de Recursos Naturais, 7 (1/4): 113-139.

Teixeira, C. V.; Mantovani, W. 1998. Vegetação na borda de um fragmento florestal na área metropolitana de São Paulo, SP, Série Técnica IPEF, 12 (32): 133-148.

Teixeira, L. A. G.; Machado, I. C. 2004. Sabicea cinerea Aubl. (Rubiaceae): distilia e polinização em um fragmento de floresta Atlântica em Pernambuco, Nordeste do Brasil. Revista Brasileira de Botânica, 27 (1): 193-204.

Vicente, A. 1999. Levantamento florístico de um fragmento florestal na Serra de Itabaiana, Sergipe. Dissertação de Mestrado, Universidade Federal Rural de Pernambuco, Brasil, 113pp.

Vidal, E.; Viana, V. M.; Baptista, J. L. F. 1998. Efeitos da exploração madeireira predatória e planejada sobre a diversidade de espécies da Amazônia oriental. Revista Árvore, 22: 503-520.

Vidal, W. N.; Vidal, M. R. R. 2000. Botânica: Organografia quadros sinóticos ilustrados de fanerógamos. $3^{\mathrm{a}}$ ed. Imprensa Universitária, Viçosa, Brasil, 114pp.

Vieira, G.; Hosokawa, R. T. 1989. Composição florística da vegetação da regeneração natural, 1 ano após diferentes níveis de exploração de uma floresta tropical úmida. Acta Amazônica, 19: 401-413.

Werneck, M. S.; Franceschinelli, E. V.; Tameirão-Neto, E. 2000. Mudanças na florística e estrutura de uma floresta decídua durante um período de quatro anos (1994-1998), na região do Triângulo Mineiro, MG. Revista Brasileira de Botânica, 23 (4): 401-413.

Zickel, C. S. 1995. Fitossociologia e dinâmica do estrato herbáceo de dois fragmentos florestais do estado de São Paulo. Tese de Doutorado, Universidade Estadual de Campinas, Brasil, 125pp.

Zickel, C. S.; Almeida Jr., E. B.; Medeiros, D. P. W.; Lima, P. B.; Silva, S. L. S.; Santos-Filho, F. S.; Lima, L. F.; Souza, T. S. M.; Vicente, A.; Souza, A. C. R. 2008. Espectro de dispersão de diásporos e distribuição das espécies de sub-bosque em três áreas de floresta atlântica (lato sensu) no Nordeste do Brasil. In: Moura, A. N.; Araújo, E. L. \& Albuquerque, U. P. (Orgs). Biodiversidade, potencial econômico e processos eco-fisiológicos em ecossistemas nordestinos. v.1. Comunigraf, Recife, p.131-157. 